\title{
Dynamics of vascular branching morphogenesis: The effect of blood and tissue flow
}

\author{
Thi-Hanh Nguyen, ${ }^{1}$ Anne Eichmann, ${ }^{2}$ Ferdinand Le Noble, ${ }^{3}$ and Vincent Fleury ${ }^{4, *}$ \\ ${ }^{1}$ Laboratoire de Physique de la Matière Condensée, Ecole Polytechnique 91128, Palaiseau, France \\ ${ }^{2}$ Collège de France, Laboratoire de Médecine Expérimentale, Inserm U36, 11 Place Marcelin Berthelot Paris 75231, France \\ ${ }^{3}$ Laboratory for Angiogenesis and Cardiovascular Pathology, Max Delbrück Cenrum für Molekulare Medizin, Robert Rössle Strasse 10, \\ 13125 Berlin-Buch, Germany \\ ${ }^{4}$ Groupe Matière Condensée et Matériaux-UMR 6626, bâtiment 11A, Université de Rennes 1, 35042 Rennes, France
}

(Received 3 May 2004; revised manuscript received 17 November 2005; published 14 June 2006)

\begin{abstract}
Vascularization of embryonic organs or tumors starts from a primitive lattice of capillaries. Upon perfusion, this lattice is remodeled into branched arteries and veins. Adaptation to mechanical forces is implied to play a major role in arterial patterning. However, numerical simulations of vessel adaptation to haemodynamics has so far failed to predict any realistic vascular pattern. We present in this article a theoretical modeling of vascular development in the yolk sac based on three features of vascular morphogenesis: the disconnection of side branches from main branches, the reconnection of dangling sprouts ("dead ends"), and the plastic extension of interstitial tissue, which we have observed in vascular morphogenesis. We show that the effect of Poiseuille flow in the vessels can be modeled by aggregation of random walkers. Solid tissue expansion can be modeled by a Poiseuille (parabolic) deformation, hence by deformation under hits of random walkers. Incorporation of these features, which are of a mechanical nature, leads to realistic modeling of vessels, with important biological consequences. The model also predicts the outcome of simple mechanical actions, such as clamping of vessels or deformation of tissue by the presence of obstacles. This study offers an explanation for flow-driven control of vascular branching morphogenesis.
\end{abstract}

DOI: 10.1103/PhysRevE.73.061907

PACS number(s): 87.18.La, 87.18.Bb, 87.19.Rr

\section{INTRODUCTION}

Understanding the development of vessels in developing organs or tissues is an important endeavor in the context of developmental biology as well as in regenerative medicine, graft and transplant surgery, tumor neo-angiogenesis, etc. However, the development of a complete vascular system is difficult to understand, because there are self-organization rules, on the one hand, and very deterministic instructions which seem to position vessels exactly on the other hand. For example, the position of the dorsal aortas and of the navel is very deterministic, identical in all individuals, while the shape of blood vessels in the extra-embryonic organs or around tumors has a quite pronounced stochastic component.

In order to understand and model the physical rules of self-organization, we have addressed in detail the development of the vasculature in the simplest of all organs of the chicken: the yolk sac. All vertebrate embryos develop with extra-embryonic organs, such as the human placenta or the chick yolk sac $[1,2]$. In the latter, blood vessel formation is easy to study by in situ microscopy.

However, development follows a temporal sequence and it is necessary to recall a few facts in order to understand the boundary conditions of this problem. The very early avian embryo (first day) has the shape of a flat disk. (In mammals it has the shape of a ball, but the embryo develops from a discoidal section of that ball.) During the first day after being laid, there exist cellular motions ("gastrulation") which will

\footnotetext{
*Corresponding author. FAX: 00-33-2 2323 6717. Electronic address: vincent.fleury@univ-rennes1.fr
}

modify the shape of this disk. These motions have been modeled in detail recently [3]; they lay down very simply the boundary conditions for the moment when the model presented here starts to be relevant (second day of development). We shall then first present the establishment of these boundary conditions, from a physicist's point of view. From this point of view, the transition from a disk towards an embryo which has an extra-embryonic organ is understandable. Next, we shall summarize the biological observations. Afterwards we go into the physical model. We present two kinds of physical models: finite-element calculations of localized phenomena and Monte Carlo simulations of the global architecture, which incorporate local features as simple Monte Carlo rules. In the end, this article shows that diffusion-limited aggregation growth [4] with a few additional rules allows one to model realistic vascular patterns.

\section{INITIAL LANDSCAPE INSIDE THE EGG}

\section{A. Formation of the yolk sac}

In summary, initially, the embryo is a disk. The south "hemidisk" moves and contracts, and the disk takes the shape of a figure 8 [3] (Fig. 1).

The contraction of the disk induces a tensile stress on the extra-cellular matrix [3]. This provokes a fissure through which the top layer of the hemidisk ingresses. This fissure nucleates in the center (hyperbolic point) of the flow. The tissue which ingresses will form the yolk sac (avian equivalent of the mammal placenta). As the cells move underneath, they induce a compressive stress which relieves the tensile stress, because they move in opposite directions as the cells 

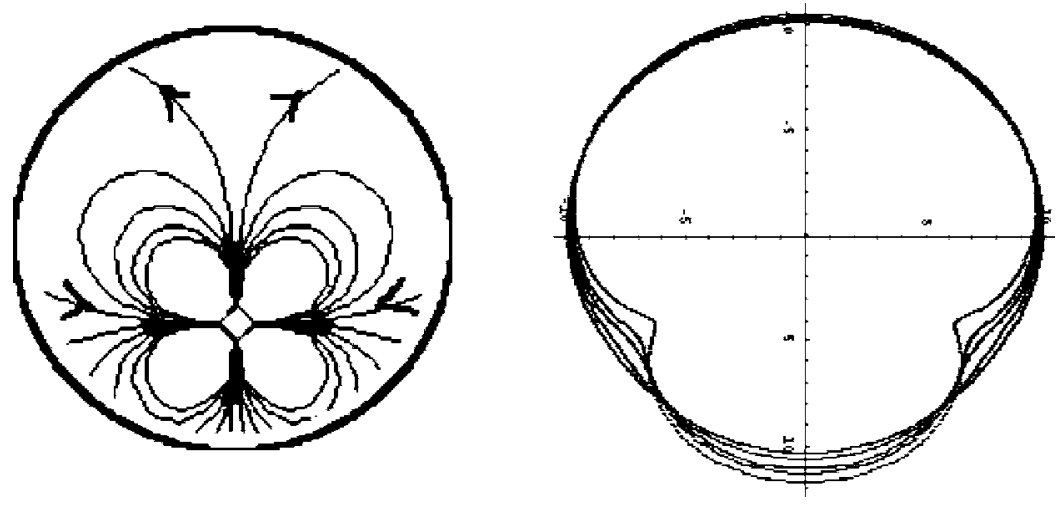

FIG. 1. Progressive contraction of the southern hemidisk (Stokes approximation) transforms the initial blastodisk into a figure 8 (from Ref. [3]). Left, the contraction-vector field lines. Right, the progressive deformation of the disk in the field given to the left. The motion of cells towards the hyperbolic point (the center of contraction) creates a tensile stress on the extracellular matrix on which cells move.

on top. Then, the crack closes and the central part of the eight folds, as the ingressed tissue moves away from the folds (Fig. 2). As it moves away, the yolk sac extends in the form of a disk of tissue, which surrounds the embryo.

As the motion continues, the compressive stress, after closing the crack, folds the central part of the figure 8 , such that crack closure is followed by the formation of the embryo body (in that spatiotemporal order). These events are a consequence of the same physical in-plane stress. The folds actually form the embryo. The folds are in the middle of the disk, and the yolk sac surrounds the folds. The central tubes of the folds form the heart, the guts, and the neural crest.

\section{B. Situation when the work presented here begins}

The initial landscape in the early avian egg, for our purposes, is therefore composed of three regions (Fig. 3). First region, right in the center along the vertical axis (with the convention of Fig. 1) the embryo, composed of elongated folds; second region, surrounding the embryo, the tissue of the initial blastodisk, still two dimensional (2D), which did not ingress and which did not fold (it is the part which has a figure-8 boundary in Fig. 3; it is called zona pellucida); and third part, the tissue which ingressed, which has become the

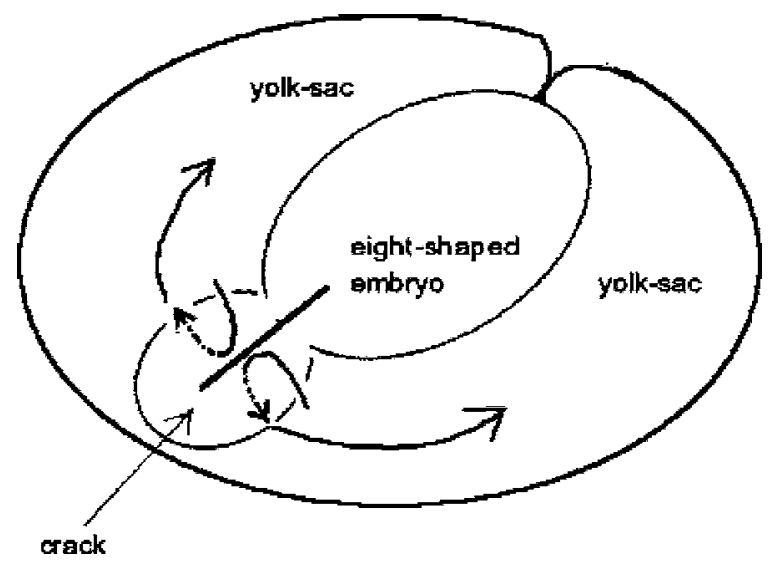

FIG. 2. Scheme of the ingression. The flow of cells in the blastodisk creates a tension which opens a crack. Cells ingress in the crack. Cells which ingress make a $U$ turn (in the vertical plane), move away from the crack, and form the large disk around the figure 8 , which is the yolk sac. The crack closes because the stress becomes compressive. yolk sac and which takes a circular form as it migrates away. This tissue is composed of a top layer (called ectoderm), a middle layer (called mesoderm), and a bottom layer (called endoderm). The formation of vessels occurs in the middle layer, about $80 \mu \mathrm{m}$ thick.

Now, the initial flow. Two folds of the embryo become the two tubular heart rudiments. These pump the blood from the anterior part and send it towards the posterior part, inside the body embryo, through the dorsal aortas. At the position of the navel, the dorsal aortas "all of a sudden" turn at right angles. The origin of this rotation is explained by the gastrulation motion: around the contraction center (Fig. 1), the force field in the disk is hyperbolic, and hence the mosaic of cells is progressively deformed into a cross, which is apparent in the shape of the vessels, which turn at right angles around the navel [5].

\section{Texture of the yolk sac}

In addition, there is a structural aspect (Fig. 4). In the yolk sac, the cells form an almost random medium because during the ingression through the crack they separate from each other [6]. In this extra-embryonic organ, a lattice of

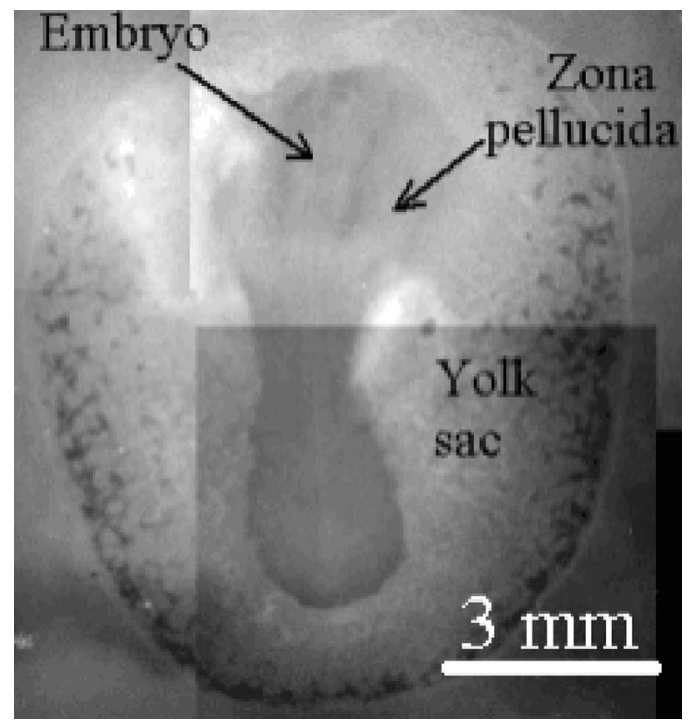

FIG. 3. The initial landscape inside the egg, at the moment when our work starts (2.5 days). The very first blood islands are visible along the edge of the yolk sac. 

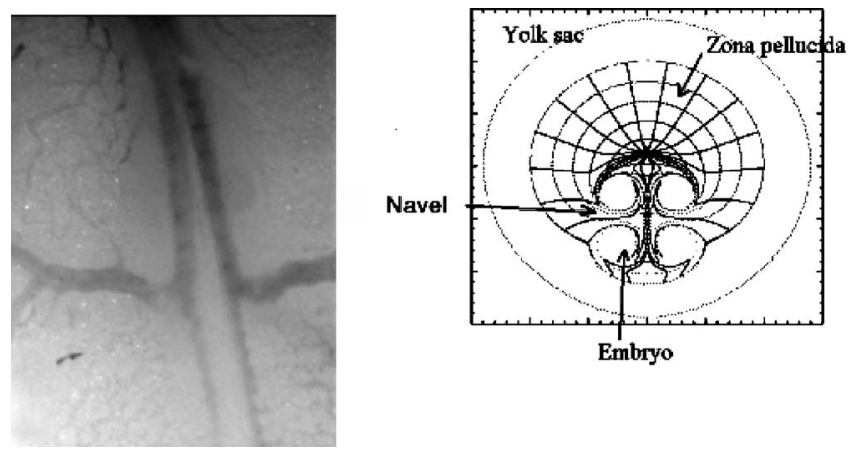

FIG. 4. Aspect of the "navel" of the chicken. The aspect of the dorsal aortas in the region of the navel is obtained by transporting in the "flow" of Fig. 1 the mosaic of the embryo [5]. In the center of the embryo, between arm and leg rudiments, one finds a turn at right angles of the mosaic (from Ref. [5]). This forms a template of vessels in the region of the navel. This gives us the boundary condition for the arterial outflow: two rudiments of arteries oriented to the right, for one, to the left, for the other (convention of Fig. 1).

small blood islands forms, called a capillary plexus [2,7]. Formation of a developed branching pattern is a matter of how this initial capillary plexus "matures," or "prunes" [7]. We address in this article the physical aspects of this pruning: how a large branching vascular tree forms, from an initial lattice of small capillaries. These physical aspects contain mainly two contributions: the effect of the flow and the effect of the tissue expansion. In a given organ, the capillary plexus may have a specific shape, inherited from deformation of the tissue; then, the pruning process has to be performed on that shape. The influence of the initial shape of the capillary plexus may define the position of the main vessels [8]. We assume here a uniform lattice of capillaries in the (circular) yolk sac.

A very concise summary of our findings is the following: growth by shear stress plus displacement of vessels by interstitial tissue pressure suffices to generate realistic blood vessels. Growth by shear generates fractal-like branching patterns akin to DLA trees, while lateral displacement stabilizes and smoothens the trees along steepest stress-gradient lines. An additional important fact is that most capillaries disconnect from vessels. This is how the initial tissue "sponge" forms tubular vessels.

\section{BRIEF RECOUNT OF WHAT IS OBSERVED IN VASCULAR DEVELOPMENT}

In this part, we summarize the experimental facts which we do need for our purpose.

\section{A. Primary and secondary circulation stages}

At the start of heart beat, blood is pumped out through the rudiments of arteries into the yolk sac and returns to the heart through the rudiments of the cranial and caudal veins [Fig. $5 \mathrm{~A}]$.

This is called the primary circulation stage in biology $[2,7,9,10]$. After day 2.5, two new venous openings occur close to the vitelline arteries and the flow pattern changes; (a)
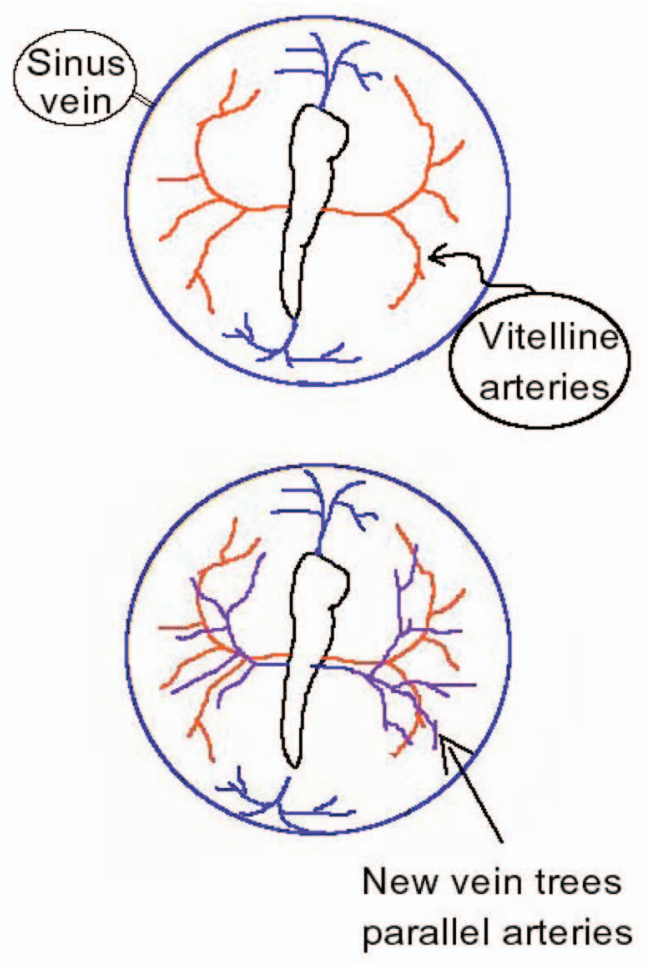

(b)

(c)

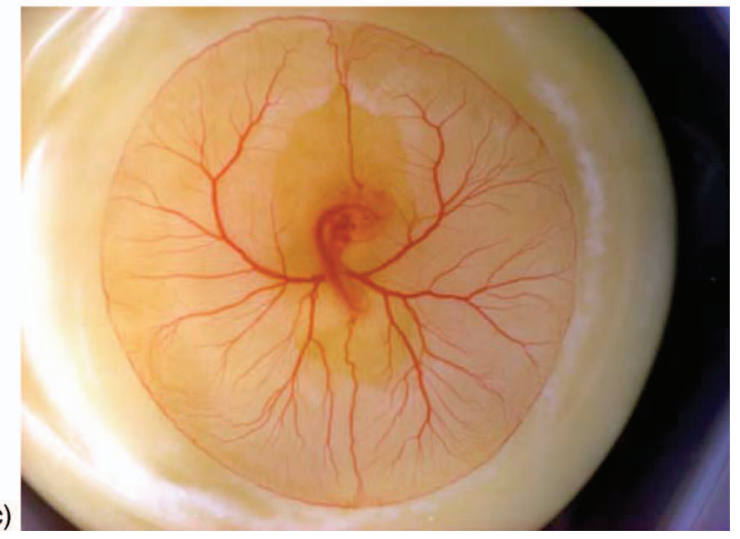

FIG. 5. (Color) (a) Scheme of the primary circulation stage. Veins are in blue, arteries in red. (b) Scheme of the secondary circulation stage. (c) The true yolk sac, in the final state which we wish to model.

instead of following arteries and veins in series, the flow rapidly functions in parallel [Fig. 5(b)]: the venous pattern closely follows the arterial pattern, so that two venous trees come out of the embryo close to the already existing vitelline arteries. This is called the secondary circulation stage in biology $[9,10]$.

In the primary circulation stage, the geometry is completely 2D (of course, vessels have a diameter, so they are $3 \mathrm{D}$, but all vessels are in the same plane and do not cross), while in the secondary circulation stage, the geometry is partly 3D: the pattern is quite flat, but vessels of two types may cross each other. Several remarkable features of this secondary circulation stage must be stressed: first of all, near the embryo, where vessels are quite large (up to $100 \mu \mathrm{m}$ in diameter), blood does not flow directly from arteries into 


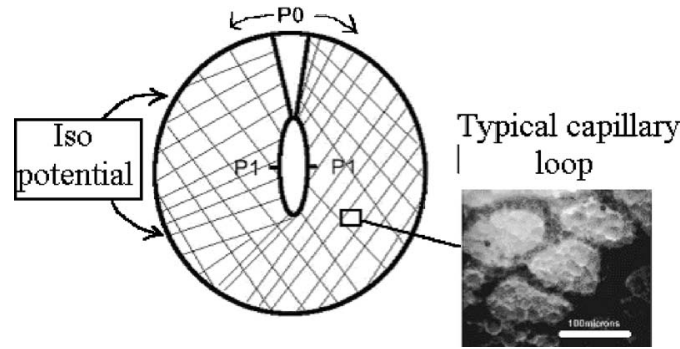

FIG. 6. Boundary and pressure conditions at start of the problem from a physicist's point of view. There is one large circular vein, isopotential at low pressure. There are two arterial rudiments at high pressure (P1). There is a random lattice of capillaries in between the embryo and the large vein. Right, an image of the true capillary lattice.

veins (no shunts), although least-resistance arguments should imply such a direct route. The arteries and veins are antiparallel: the flow is opposite in trees which follow the same pattern.

In the primary as well as in the secondary circulation stage, circulation of the blood leads to a remodeling of the capillary plexus into branched arteries and veins. Arteries and veins appear a few hours after perfusion. Arteries form downstream the rudiments of arteries, centrifugally from the embryo, and upstream the rudiments of veins, centrifugally too.

In addition to this description, there exists a large circular vessel, called the sinus vein [Fig. 5(a)], which surrounds and limits all the region of vessel formation; this vessel lines the external boundary of the growing yolk sac. This circular vein gets connected to the embryo by two anterior veins and, eventually, by one posterior vein.

In conclusion, the geometrical boundary condition and pressure conditions, from a physicist's point of view, are as shown in Fig. 6.

\section{B. Mechanism of vessel remodeling}

It has long been recognized that flow is essential to the formation of vessels [10]; as early as 1893, Thoma had the intuition that vessels followed a least resistance path of flow, although this was never actually proved, and that vessels which experience a high flow widen. Old models of vascular morphogenesis, based solely on molecular diffusion (in the manner of Turing) have been very seriously questioned [11]; they cannot account for the very complex interlace of arteries and veins. Also, very simple mechanical actions have enormous consequences on blood vessel architecture (see below), although these actions hardly interfere with molecular diffusion. Other models of vascular morphogenesis, based on shear stress and transmural pressure, have been put forward [12-15]. Adaptation to mechanical forces has for long been considered to play a major role in arterial patterning and to guide gene activation, but numerical simulations of vessel adaptation to hemodynamics, including very refined biomechanical feedbacks ("mechanotransduction"), so far have failed to predict any realistic vascular pattern. The most complex models make use of lattices of resistances whose resis- tances change as a function of the current passing through them. Therefore, these models make extensive use of Kirchoff's law for resistance networks to model the vessel adaptation as a function of time. In these models, starting from a lattice of bonds $i j$, connecting vertices $i$ to vertices $j$, incremental variations of resistances are performed numerically; then, the flow is recalculated and so on, step by step, until a stable vasculature is presumably obtained. However, these models fail to produce any realistic vascular pattern. Grier and Mueth proposed a model [16] in which the permeability of the vessels plays an important role, but again, the model does not give any realistic pattern, and moreover, the origin of vessel permeability is not discussed. More recently, Fleury and Schwartz [17] proposed that blood vessel adaptation of the capillary plexus is akin to the diffusion-limited aggregation model, in its dielectric breakdown version $[17,18]$. In this model, the growth of the vasculature is analogous to the propagation of a cluster which grows in the direction of high gradient of a Laplacian field. This analogy is easy to understand: inside a small cylindrical tube, or between plates separated by a thin gap, the flow speed $v$ has a Poiseuille-like profile [19]:

$$
v=-\left(\pi R^{2} / 2 \mu\right) \operatorname{grad} P\left(1-[y / R]^{2}\right)=V_{\max }\left(1-[y / R]^{2}\right),
$$

where $y$ is the distance to the center, $\mu$ the viscosity, and $r$ the radius. The speed is zero on the walls and maximum at the center, and grad $\mathrm{P}$ the gradient of pressure. The flow rate is

$$
Q=\left(\pi R^{4} / 8 \mu\right) \operatorname{grad} P .
$$

So the conductance of a vessel of fixed length (ratio of pressure difference over flux) varies as the fourth power of its radius.

The shear stress at the wall is given by

$$
\tau=-\mu \partial v / \partial y=(2 / R) V_{\max }
$$

which shows that the shear stress diminishes with increasing radius. When considering a lattice, the gradient is only the pressure difference between neighboring vertices $i$ and $j$, divided by the lattice unit which fixes the length of individual strands in the plexus. Because of incompressibility, the pressure satisfies the Laplace equation in the plexus. The shear stress on the tube wall is proportional to the speed in the tube for a given constant diameter of the tube; the shear stress is then proportional to the pressure drop between the vertices. "Growth" of the vessel amounts to a replacement of a capillary bond $i j$ of diameter, say, $r$ by a segment of blood vessel of diameter $R$, with $r \ll R$. It is assumed in biology that endothelial cells feel shear stress and transmural pressure and, hence, react by reorganization to enlarge the vessel. The constitutive equation of this enlargement is not known. However, in the simplest case where the enlargement is proportional to shear stress, the speed of forward progression of the large tube is proportional to the speed of flow in the nearest small tube forward. The new segment of diameter $R \gg r$ is much more conducting than the small vessel. Therefore, the 
vessel pattern may be considered as very conducting, which means equipotential in terms of pressure.

The progressive replacement of small vessels by larger vessels is then formally identical to the propagation of a diffusion-limited aggregation (DLA) cluster in the dielectric breakdown version $[4,20]$, the aggregate being the vessel tree and the lattice around being the capillary plexus. The flow is supposed to flow between a high-pressure point and a lowpressure point kept at constant pressure ("constant potential" in the DLA picture), these boundary conditions are fixed by the heart. It has been shown that this model leads to easy self-organized growth of vessels quite reminiscent of blood vessels [17]. It has even been shown that this model leads to a simple interlace of arteries and veins in 3D if capillary disconnection is included [18] (see below).

However, although convincing in terms of selforganization, adaptation, and phylogeny, none of these models lead to truly realistic vessels. What is wrong with these models? For all these models, the vessels are nondeformable static tubes, except for the diameter variation. This, we believe, is the error. In order to form realistic vessels, one needs to take into account the deformability of vessels. This deformability leads to several new features. Especially, the deformability at junctions leads to a closing and opening of tubes at the junctions, which explains the very origin of vessels (see below). The expansion of the tissue leads to a progressive displacement of the tubes which explains why they eventually form straight segments. The deformability of dead ends leads to a regrowth and reconnection of dangling sprouts to existing vessels, which explain the crossing of vessels ("dead ends" of vessels are formed when a capillary is disconnected from an artery or a vein, so formation of a true vessel is concomitant with formation of multiple sprouts).

Incorporation of these three features into a simple physical model allowed us to create and explain complex and realistic arterial-venous trees. Moreover, the model can predict the consequences of localized alterations of blood flow, such as arterial occlusions, on the overall pattern of arteries and veins. This model improves our understanding of neonatal anomalies, disease-related abnormal vascular patterns such as tumor vasculatures, and development of collateral circulations in ischemic diseases and drug- or growth-factorinduced changes of vascular patterning.

\section{DETAILED DESCRIPTION OF THE NEW FEATURES}

\section{A. Detailed description of capillary disconnection}

To ensure embryo growth and nutrient and oxygen uptake, blood flow has to be distributed to the most distal (note that in biology distal means away from the body or organ center) parts of the capillary plexus (i.e., the exchange area). Only small areas of tissue can be sustained with diffusion across capillaries only. This is why larger tubes are needed, to bring flow directly to distal parts. High-magnification video microscopy has shown that this distalization of flow is achieved by disconnection of small arterial side branches from larger arteries that form the initial "flow highways," Figs. 7-9 and

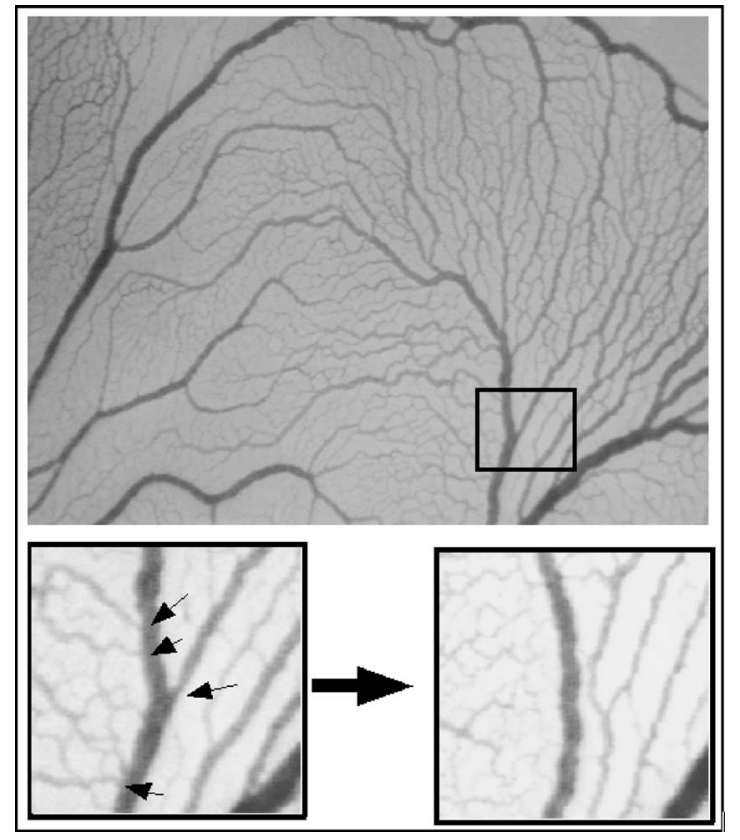

FIG. 7. Examples of small vessel disconnection (arrows), including capillaries, and even one venule. The capillary-free zone appears clearly on the left of the main venule (bottom right) and also around the top-left arteriole.

Ref. [9]. Without this disconnection, vessels would simply not form.

The paradox of vascular morphogenesis is, then, that actually a considerable number of vascular openings have to be closed, and not opened, to reach a functioning vasculature, and even to just make a tube. A typical chick vessel, seen from end to end, shows that the number of lateral connections to capillaries is much smaller in proximal parts than in distal parts (Fig. 8). In distal parts, the vessel is merged into the capillary plexus. In proximal parts, the vessel is a tubular entity in itself, with almost no lateral orifices. As the disconnection happens, the arteries see more flow (because disconnection interrupts "leakage") and they become larger. The point at which a given lateral capillary gets disconnected is

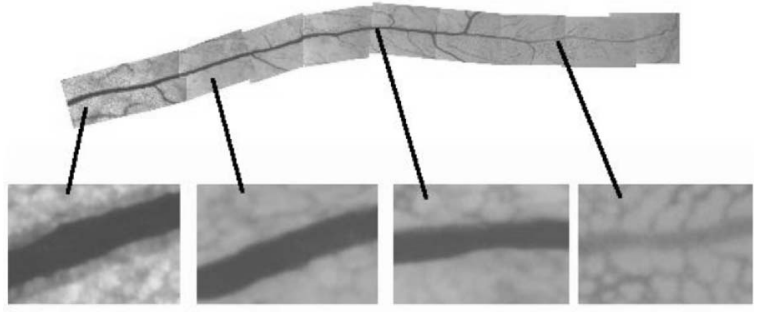

FIG. 8. Morphology of a chick yolk sac vessel, by day 3.5, seen from end to end (approximately $2 \mathrm{~cm}$ ), showing that the density of connected capillaries is larger in distal parts than in proximal parts. In the first two segments shown, there are simply no connections to the capillary plexus; in the third, they are hardly visible, if any. Perfused capillaries connected to the vessel are found mostly in distal parts (fourth segment). In proximal parts, one would be able to cut vessels and separate them from the mesoderm; distal parts, vessels are intimately connected to the tissue by capillaries. 

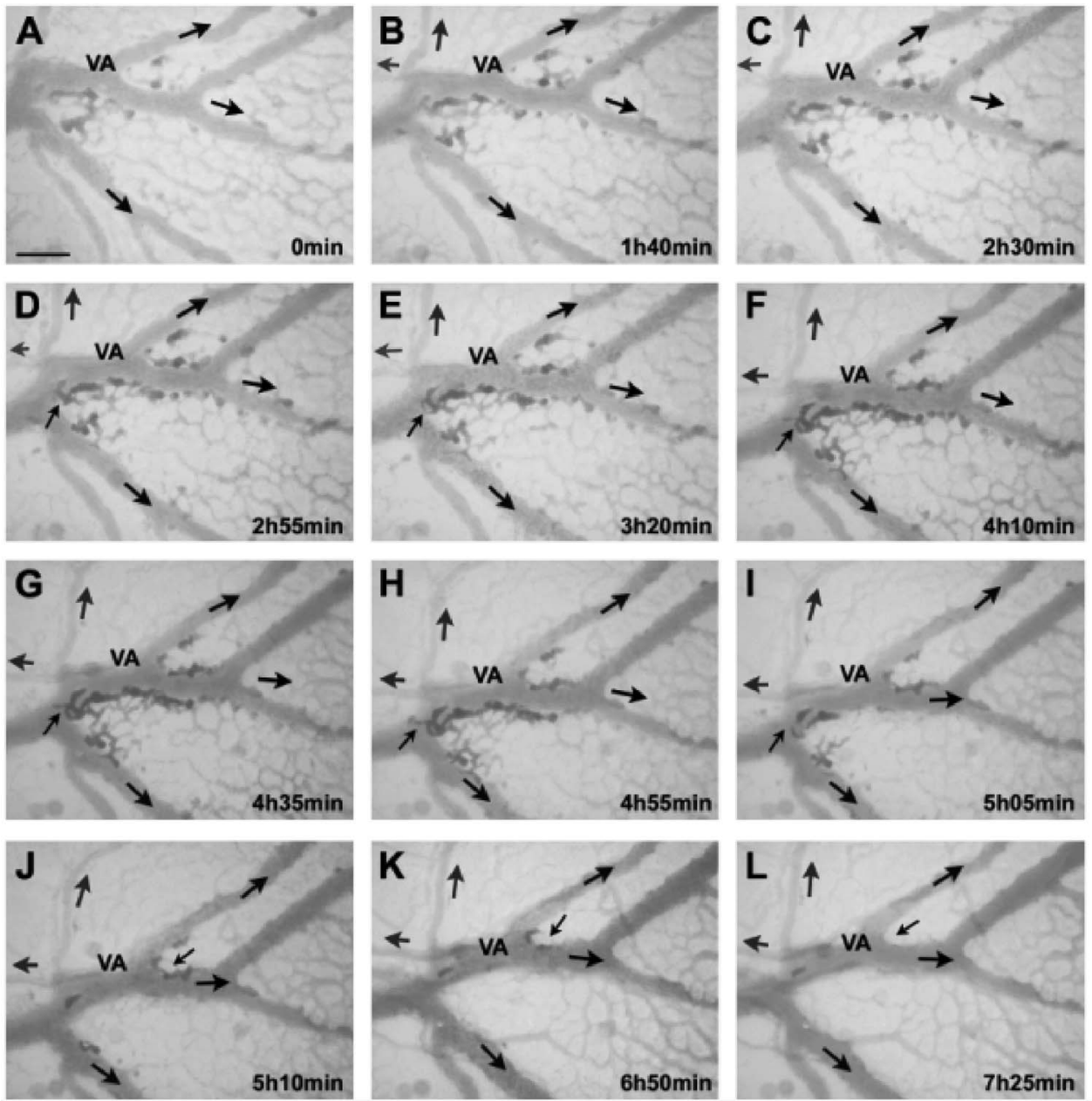

FIG. 9. Time lapse imaging of capillaries which are disconnected from arteries, then reconnected to veins, and again perfused (reprinted from Ref. [9]). This image shows first a vitelline artery [VA in (a)] with several capillary loops which are connected to it; others are already disconnected. From (a) to (f), more capillary loops are disconnected. As a consequence, the dead ends appear as stagnant blood islands, which lie along the artery, but are no longer in contact with the artery lumen. The dead ends behave as sprouts; especially, from (f) to (i), black arrows show a dead end which succeeds to grow over the artery and to reconnect to the other side. As an instantaneous consequence, the blood is flushed from the stagnant islands and flow is resumed in the strands along the artery. Note that the direction of blood flow in these strands is reversed. Now, venules can form in the capillaries which are disconnected from the artery and can be incorporated in the venous bed, in the proximal to distal order, following centrifugally capillary strands which are oriented parallel to the arteries and growing in the direction of arterial tips.

very localized at the junction with the bigger tube; it does not require a shrinkage of the entire tube between two vertices. As a consequence of the disconnection, large capillary-free zones appear around the arteries [9,17,21,22], but not, or much less, around the veins. These large capillary-free zones surrounding main vessels are also known to exist in the brain [23]. In this picture, the resistance of the vertices where the vessels may be strangled depends on the ratio between the flow in the bond $i j$ and the total flow passing through the vertex $i$. Therefore, it is entirely different from the progressive narrowing of a bond $i j$ as a function of the flow passing through the bond $i j$ itself. This is how true tubes without leakage form from a spongy percolating lattice. Now, what happens to the dangling bonds formed by the process of disconnection?

\section{B. Detailed description of capillary reconnection}

Disconnected vessels often do not regress. The disconnected arterial segments (dangling dead end) subsequently behave as sprouts, Fig. 9 .

These sprouts are still connected to the arterial tree through more proximal connections. High-magnification microscopy has shown that hemodynamic oscillations (systolediastole beat) can been observed in the dead-end lumenized sprouts. Although they experience no average flow (dead end), these sprouts grow and swerve around the main arterial segment from which they were disconnected, and they reconnect to the plexus on the other side of the vessel and become essential to fashion the growth of embryonic veins. As a consequence of these processes, the vascular plexus now has a 3D configuration, with possible crossing of vessels, after 


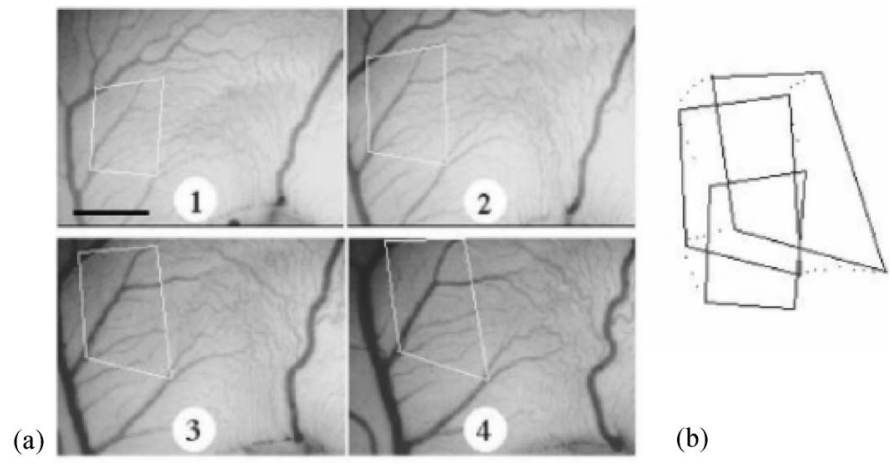

FIG. 10. Vessel displacement is readily observed on time-lapse movies [(a), photos 1-4]. The photo sequence shows a developing part of the chick embryo yolk-sac vasculature between days 2 and 3. In photo 1 four branch points are selected and followed over time; photos 2-4 show the fate of this region over a period of time of $8 \mathrm{~h}$. We visualize the differential growth of the tissue, by overlaying a polygon, whose corners are at four chosen vessel bifurcations, considered as absolute reference points. We follow the rectangle during time (panel right). It is observed that the rectangle is deformed and displaced. The motion is not a mere homothetic dilation, and it is completely self-organized by the branches as they grow.

these sprouts have themselves enlarged. This is how a $2 \mathrm{D}$ plexus becomes $3 \mathrm{D}$, and this is why vessels crossing each other may give a false impression of being connected.

\section{Detailed description of vessel displacement}

Simple physical deformations of the tissue surrounding the blood vessels can change the position of the branched arteries and veins within the tissue dramatically. In vivo observations over time of selected vessel branch points showed that vessels are displaced relative to each other (Fig. 10).

This is the result of growth in-between vessels and vascular stretch, since vessels as such do not have motility. There exists a stretch component coming from the inside due to flow and one from the outside due to tissue expansion. The displacement of vessels tends to make tortuous vessels straight, and it strengthens the angles. Conversely, a lack of stretch and displacement tends to make vessels more tortuous, as evidenced by stretch relief experiments (data not shown). Vessels are physically moved over time over considerable distances, but not according to a mere homothetic dilation. The force exerted locally is self-organized, depending on the local force exerted by each vessel and each point in the tissue.

\section{NUMERICAL SIMULATIONS OF DISCONNECTION AND RECONNECTION}

\section{A. Modeling of disconnection}

Disconnection of side branches occurs exactly at the point of bifurcation, without affecting the remaining part of the side branch $[7,9]$. Our ansatz is that the bifurcation is as much prone to adaptation as the tube between two vertices. We thus calculate the deformation exerted by the blood flow on the blood vessel wall, right at the point of bifurcation. In order to calculate this adaptation, we assume that the tissue forming the vessel and its surroundings will adapt to flowinduced deformation by a simple elastoplastic adaptation. If we couple flow inside the vessel with elastic deformation outside the vessel (fluid-structure interaction) [Fig. 11A], we observe that closing of side branches is a typical behavior which will occur in a narrow region around the interconnection, where the assumption of Poiseuille's flow (which is only valid for straight, not branching, tubes) breaks down, leaving the rest of the tube almost unchanged. The calculation was done for an isolated bifurcation, with a given flow velocity in the larger and in the smaller vessel. In principle, the pressure and flow field should be entirely recalculated self-consistently, taking into account the vessel architecture upstream and downstream. However, we are interested here only in the generic microevent of closure, rather than in the consequences at the architecture level, which will be addressed below.

Closing of side branches is an irreversible phenomenon, which disconnects capillaries right at the connection. The effect of this morphological change is indeed that blood flows towards more distal regions (where it is needed in the exchange area) while vessels become true tubes without "leakage" and with increased flow. Since the process repeats itself in a proximal-to-distal progression during development, it permits outgrowth of the arterial tree, preventing the formation of arterial-venous shunts. In the same time, systematic closing of orifices transforms the random plexus into true tubular entities in series.

Still, the pruning process does generate bifurcation points, so not all side branches are chopped off. Our simulations show that the local initial closure rate is controlled by the flow direction, the amount of flow, and, most importantly, the branching angle [Fig. 11(b)] (the absolute pressure also matters, but this parameter was not varied in these calculations). At the venous side, where blood flows from the side branch into the main venule, our elastoplastic adaptation model predicts a smaller closure rate and even the opposite phenomenon: a widening of the venous side branch above a threshold of flow speed. For arteries, there also exists a domain of parameters in which small vessels open up instead of closing, thus allowing the formation of bifurcation points, but this domain is much smaller than for veins. This is congruent with observations of the microcirculation in vivo in which terminal arterioles tend to be less numerous than the postcapillary venules.

Examining the effects of branching angle shows that closing of side branches lateral to arteries occurs in this approximate model, at angles close to $75^{\circ}$ or higher, while small bifurcating angles $\left(<60^{\circ}\right)$ leave side branches open in a wide range of speeds. This explains why only a subset of side branches are selected and why certain bifurcating angles are directly selected by the flow. Therefore, our model suggests that the flow selects by self-organization of a dielectric breakdown model a tree which follows a subset of the capillary plexus which has the proper angles already. This subset is itself remodeled progressively, so that among the strands of this subset, only the ones experiencing enough flow will enlarge. Of course, in our calculation, the flow is imposed in 


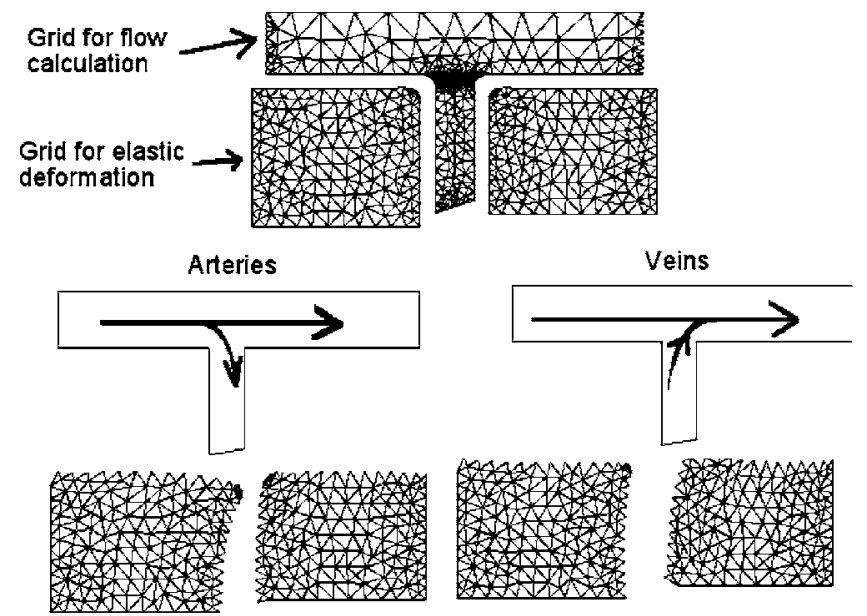

(a)

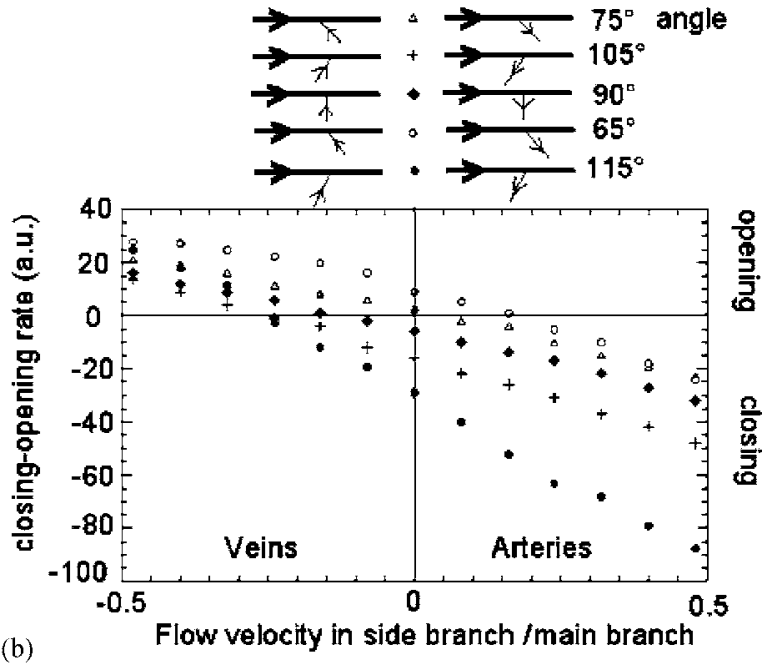

(b)

FIG. 11. (a) (Left) We have studied the flow induced elastoplastic deformation in the region where a side branch is connected to a larger main branch using a physical model. In the model we have solved the Navier-Stokes equation in a cavity representing the connection (a), for both arteries and veins. As suggested by in vivo observations, we started with a high flow velocity in the main branch [horizontal, in (a)] and a smaller flow velocity in the side branch. The flow velocity was assigned positive when separating from the main artery to a lateral smaller arteriolar capillary (if the two flows separate at the connection Vy is oriented downwards in the lateral vessel) and negative in the other case (if the two flows converge at the connection, the flow Vy in the lateral venous capillary is oriented upwards). We also did the calculation for a flow velocity equal to zero (stagnant flow-e.g., the side branch is a dead end as is the case for an angiogenic sprout). Once the flow chart is computed, we use the pressure and velocity along the boundary in order to calculate the elastic deformation of the surrounding tissue. In order to find the deformation, we solve Hooke's law outside the vessel using as boundary condition the pressure and shear exerted by the flow (a). These calculations are performed with the finite-element method in 2D. This gives the incremental elastic deformation induced by the flow [two examples are given in (a), bottom]. When compared to the initial configuration, this gives either a closing (bottom left) or an opening rate (bottom right), a negative (positive) rate for the width of the side branch. (b) (Right) We plot here the "first step" or initial opening-closure rate as a function of the flow velocity in the side branch and as a function of the branching angle of the side branch. The results show the domain of parameters in which arterioles or venules either tend to remain connected or to become disconnected, as a consequence of the elastoplastic behavior of the cells at the bifurcation. The plot is therefore formed of four parameter domains [on the $x$ axis: veins or arteries corresponding to negative or positive flow velocities: on the $y$ axis, closing (negative) or opening (positive) rate]. For each angle, we find that the domain of arteriolar disconnection is much wider than the domain of vein disconnection (see text). In the arterial domain, side branches remain connected to the main branch for sharp angles only.

an isolated ideal configuration, while in a true vascular system, the entire pressure map has to be recalculated each time a vessel is modified, with possible long-range effects. However, the results given in Fig. 16, below, suffice to understand the principle of the phenomenon.

\section{B. Numerical simulation of capillary reconnection}

The second critical morphological event that needs to be addressed is how physical forces may drive the movement and sprouting of the disconnected segment over the main branch. If we consider the shear generated at a dead-end capillary facing a rigid cylinder, we see that the incentive for growth (hence the "navigation" of the dead end) in response to shear is oriented in the circumferential direction around the tube [Fig. 12(a)].

A growing sprout pushing itself forward will undergo irreversible deformation above a threshold of plasticity (yield). As a net result, the sprout navigates in between larger tubes and avoids them [Fig. 12(b)]. Therefore the same adaptation rules accepted for perfused vessel lumen explain naturally why a disconnected vessel which has internal pressure grows over and around the main arterial vessel, even in the absence of flow, and may reach and only fuse with available small vessels on the other side. Unfortunately, we were not able to complete entirely the growth of sprouts all the way around a bigger vessel due to numerical instability of the finiteelement mesh at the sharp tip of the growing sprout, after several tens of remeshing.

\section{COMPLETE STOCHASTIC MODEL}

Do the morphological features linked to deformationdisconnection, crossing with subsequent reconnection, and displacement-explain and predict arterial-venous patterning? In order to generate large-scale architectures, we will make use of a stochastic model.

\section{A. Modeling of the growth of the arterial tree}

It is classical in Monte Carlo simulations that one finds the solution of the Laplace equation by means of random walkers $[24,25]$. The pressure map in a lattice of tubes connecting vertices $i j$ is given by the concentration of random walkers passing at each point. The random walkers are launched at a point of high pressure and removed at the point 

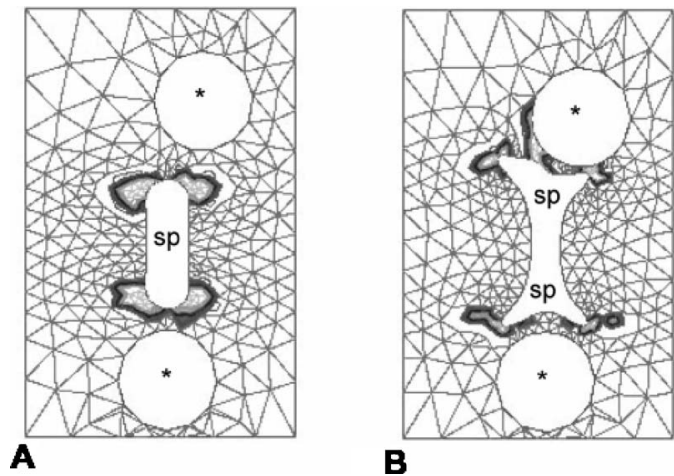

FIG. 12. Using a simple elastoplastic model, we perform iteratively the growth of a small vessel sprout (SP) or tip, which faces a tube (a). The calculation is performed in $2 \mathrm{D}$ by the finite-element method. In this figure, a segment of the small disconnected tube is seen facing two bigger tubes. We show here simultaneously (a) one tip facing a tube in a symmetrical situation [(a), bottom part] and one tip facing a tube in an asymmetrical situation [(a), top part]. Stars show the arterial lumens. Pressure is exerted from the inside of the small sprout. This creates a stress field around the tip. We select an ad hoc threshold of shear. The colored line shows the region above this threshold. Many (up to 50) elastoplastic steps of growth are performed iteratively by letting the sprout deform and by considering that only the deformation above a certain threshold is plastic (deformation for good), below the threshold the deformation is elastic. The results (b) show that the disconnected vessel preferentially sprouts out from the tips and the sprouts tend to avoid the large tubes and grow towards the most favorable region, hence swerving around the tube. This effect, however, is dependent on the magnitude of the threshold, for the elastoplasticity of the tissue and, above all, on the components of the deformation which are chosen for the plasticity level (this translates in biological terms in whether cells divide and adapt in response to tensile deformation or to shear, or to a combination of them; in this calculation, only shear is taken into account).

of low pressure. This comes from the fact that in steady state, the diffusion equation and the Laplace equation are formally identical:

$$
\Delta P=0 .
$$

This comes from flux conservation:

$$
\text { div } \operatorname{grad} P=0 .
$$

The random walk is a discrete and probabilistic version of the diffusion equation, with conservation of probability at vertices, which gives the solution of the Laplace equation, on average.

The diffusion-limited aggregation model [4] consists in growing an aggregate by letting such random walkers aggregate when they touch a seed or the already aggregated particles. The growth of the cluster is a stochastic version of the growth of a cluster in the direction of the high-pressure gradient, itself formally identical to the progressive replacement of small very resistive tubes, by large conducting tubes, in the direction of high flow. We shall therefore grow blood vessels by DLA growth. The aggregate itself is a vascular tree; it is supposed to be formed of very conducting strands, while the surrounding lattice is supposed to be composed of very resistive strands (capillaries only, which we do not represent in the images below). Therefore, in this model, there exist only two types of vessels: capillaries (the surrounding medium where random walkers move) and large vessels (the aggregate).

\section{B. Modeling of the regression and reconnection of capillaries}

In order to introduce capillary regression, we simply consider that the closing of connections occurs in the oldest fraction of existing vessels. For example, we may decide that only the $20 \%$ last formed vessels (last aggregated random walkers) are still connected to the capillary plexus. The justification of this rule is the following: it is known in diffusion-limited aggregation phenomena that the active region scales with the size of the aggregate and occupies only the external boundary ("canopy" of the tree). This in return means that the flux is much smaller ("screened") in the older parts of the tree [25]. As a consequence, we may consider as a simplification that the ratio of current inside the vessels and currents flowing out of tips goes below a critical value, inducing capillary disconnection, by the monomers which are "old enough" and with an age which scales with size. This is both because the lateral flow decreases and, in the meantime, the longitudinal flow (towards more distal areas) increases. This selects by self-organization the distal parts as the parts still connected to the plexus, which is qualitatively correct, and the dimension of these distal parts increases with the absolute size.

In order to model capillary reconnection, we fix a probability for a random walker facing one disconnected tube to jump over and connect a dangling bond located on the other side, across an existing vessel. This corresponds to considering a pressure gradient between either sides of an existing arterial tube to be the incentive for growth of a venous sprout, albeit with a difficulty to cross the tube, which is qualitatively correct.

\section{Modeling of the displacement of tubes}

It has been shown recently that the deformation of the embryo and of the yolk sac at early stages can be modeled by a slow viscous flow of the cells $[3,5]$. This flow follows a Laplacian field in the tissue, which is not the same as the Laplacian field inside the tube, which was used above. This field lies in between vascular tubes, while the previous one lies entirely inside the tubes. Inside the tubes, there is a true liquid (blood). In between the tubes, it is a plastic solid which behaves like a liquid because it is a thin layer [3]. In this solid,

$$
U=-\left(h^{2} / 12 \nu\right) \operatorname{grad} P
$$

with

$$
\Delta P=0,
$$

where $U$ is the solid flow in the middle, $\nu$ an equivalent viscosity, and $h$ the thickness.

The fact that the deformation rate in the yolk sac, in between tubes, is Laplacian allows us to introduce a new set of 


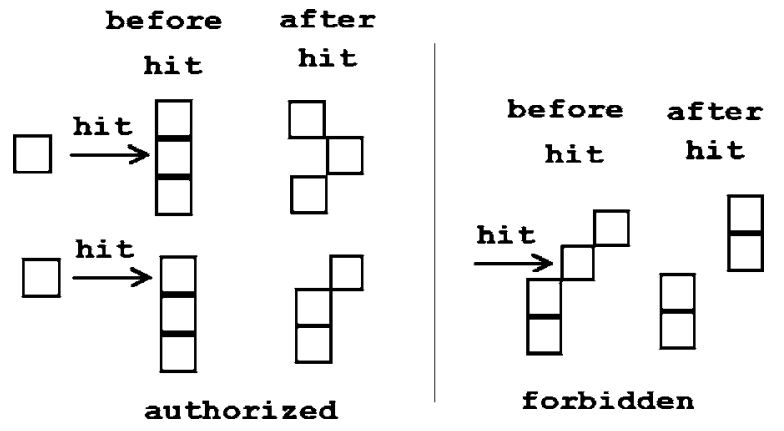

FIG. 13. In our stochastic model, random walkers model the effect of interstitial pressure. When these random walkers arrive on the tree, they shift it sideways by one pixel (two examples of hits are shown to the left), except if such a move would disconnect the tree (one example of forbidden hit to the right).

random walkers for the displacement field. This is probably the newest feature in this sort of simulation. Random walkers in the tissue correspond mathematically to pressure in the solid, and upon hitting a vessel, they shift it sideways by one pixel, because the gradient of pressure is proportional to the deformation. However, we consider that the aggregate (the vessels) cannot be disconnected (Fig. 13) by such displacements. This amounts to considering that there is a line tension to the vessels: it costs a lot of energy to interrupt an existing well-formed vessel.

Technically, we therefore launch random walkers (which we call displacement walkers), which, upon hitting the aggregate, displace the strand which they have hit if the local connectivity is maintained. Displacement walkers may be launched from everywhere, if one assumes that the yolk sac expands by catching up nutrients directly in the yolk underneath, or from the vessels only, if one assumes that expansion requires growth factors carried by the flow. Actually, we found little difference in these two assumptions at this level of description. In the simulations shown, they are launched from the vessels. Since vessels span the entire yolk sac, and since the Laplace equation has a smoothing effect, it makes little difference to launch walkers from the disk or from the branches.

When the displacement walkers reach a tip instead of a lateral side, the tip is not retracted if the flow is strong enough inside: this is simply modeled by considering that the last arrived monomers, which are the active tips which see a large flow, cannot be retracted. Other dangling tips, which have for some time not grown, are supposed to see very little flow and, hence, can be retracted if displacement walkers reach them. Therefore, this parameter models the inhibiting effect on growth of transmural pressure if the internal flow is not strong enough.

Although all the rules given above may seem complex, in the end they each describe a simple feature which is observed and which are all of a physical origin. In summary, there are four numerical parameters in this model. One parameter describing the plasticity of vessel bifurcations, which is the ratio of still connected monomers, over the total number of monomers. Second is the probability of a dangling disconnected capillary to reach out on the other side of a vessel; this parameter describes the elastoplastic regrowth of a sprout around an artery. Third is the number of very last tips which can resist shrinkage; this describes the competition between shear inside and shear outside the vessel. The final parameter is the number of displacement steps in between each growth step-i.e., the number of displacement random walkers launched - in between growth random walkers. This models the proportion of tissue expansion versus local reorganization of vessel diameters or, in other words, the interstitial tissue "viscosity" over the cell wall "viscosity."

However, several noticeable features are absent from such a simulation: the longitudinal effect of the force exerted inside the vessels, leading to intercalary extension of strands (this is absent from DLA models, which always grow by tips; however, our model contains longitudinal extension by external forces), the effect of shear inside the vessels, on the surrounding medium, and buckling instabilities of strands which could lead to meandering vessels. This cannot be treated without solving the elasticity equations finely. The effect of diffusible molecules such as growth factors or even oxygen is not treated here.

\section{RESULTS}

Integrating these features in simple Monte Carlo simulations (Figs. 14 and 15) shows that we can now grow, by self-organization, arterial and venular networks highly similar to those observed in chick embryo yolk sacs in vivo. Surprisingly, realistic architectures are obtained without having to take into account Kirchoff's law. This points to the fact that mechanical forces deform and pattern vessels, with rules which are not restricted to diameter adaptation of static lattices in response to shear and transmural pressure, as so far considered. It is necessary to gently and progressively move vessels to form a realistic vasculature.

The model reproduces and explains a considerable number of in vivo observations.

In Fig. 14 we start with a simplified version of the primary circulation stage: two venous rudiments on the cranial side (vitelline veins), corresponding to the initial situation observed in a day-2 chick embryo. Two arterial rudiments (left and right vitelline arteries) are put in the center of the embryo. The simulation generates the fusion of the cranial venular segments into a single strand, which connects itself to the sinus vein by an angular reentrance. This reentrance is typical of a geometrical feature linked to mechanical equilibrium, which cannot be obtained by resistance network adaptation. The sinus vein, and the entire yolk sac, extends stably, under the progressive deformation generated by the vessels "push." Formation of bows or loops can also be observed at a smaller scale.

The arteries display more branching vessels towards the convexity than towards the concavity, as observed. The physical explanation of this fact is simply that the Laplace equation is screened inside a concavity (small flux), while the flux is higher on a convexity of radius of curvature $R$ (being proportional to $1 / R$ ). This is again a geometrical fea- 
(a)

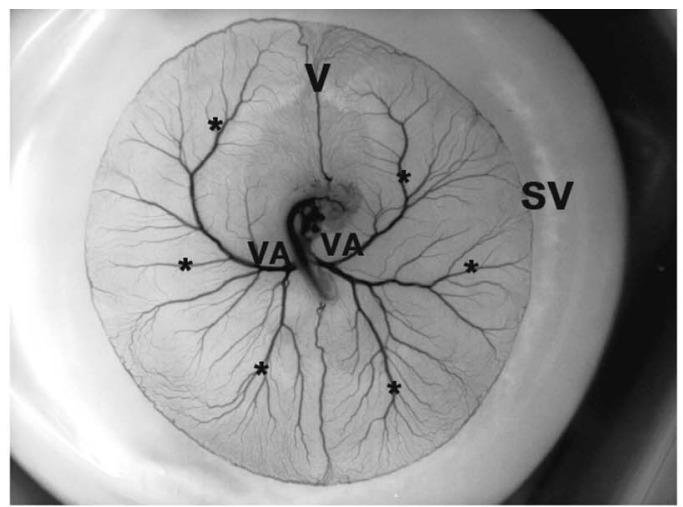

(b)

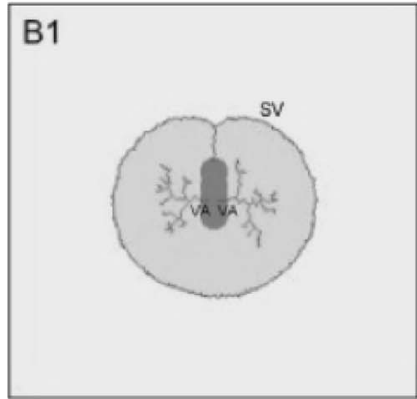

B2

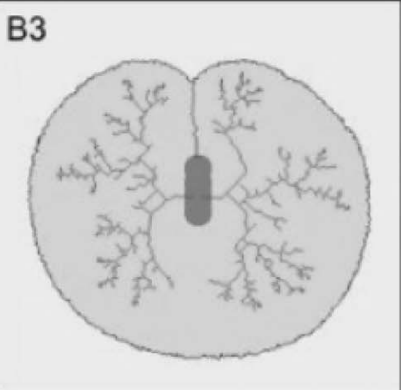

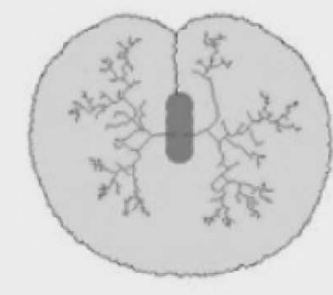

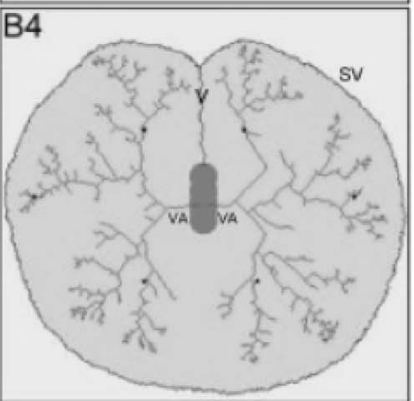

FIG. 14. (a) Typical true yolk sac. SV=sinus vein. Stars=arteries. VA=vitelline artery. V=cranial vein. The embryo is clearly visible in the center. (b) Numerical modeling by Monte Carlo simulation of the morphogenesis of a vascular tree (B1)-(B4). The model is defined by an initial boundary condition and capillary plexus (a matrix of pixels). In this simplified simulation, we put two arterial rudiments at the center and two venous rudiments at the head only (we do not include the caudal vein). Next, we use random walkers (RW) on the lattice to model diffusion fields around the vessels and hence the effect of both shear inside the tube and push outside the tubes (see text). When a growth RW hits an existing vessel, itself part of a tree, the RW is aggregated to it and the vessel extended by one pixel. Such a random aggregation is a stochastic version of capillary enlargement by adaptation to stresses; the growth of the aggregate (seen here as a growing red tree) is formally identical to enlargement of capillaries in the direction of high gradient of pressure (the vasculature grows proportionally to $-\operatorname{grad} P$; see text). When a displacement RW hits the tree, the monomer of the tree is moved sideways by one pixel. The parameters of this simulation are the total fraction of disconnected monomers, at each step, $70 \%$ of all segments. (For example, if 1000 monomers are present in the tree, the first 700 are disconnected.) The ration of displacement hits with respect to the growth hits is 4 . (This to say that four displacement random walkers are launched between each extension random walker.) The nonretractable monomers are the last $5 \%$ which have arrived. In (B1)-(B4), the arterial tree is actually surrounded by a lattice of capillaries which is not represented for reasons of clarity. Since blood flows out of arteries towards veins, the particles driving the growth of veins are launched from the arteries and vice versa. In order to investigate the remodeling of the vasculature in reaction to interstitial tissue shear, we incorporate pressure driven displacements of vessels. We add to the model a second family of RW's which, coming from vessels, move the vessels. Upon hitting a vessel, a RW simply moves the hit vessel laterally by one step. In some dead ends (tips), the motion amounts to a retraction (see text). The actual motion of vessels is the self-organized result of all these pushes. To model capillary disconnection, vessels are progressively disconnected from the plexus as they grow. In the simulation displayed here, we show only the arteries, to make the comparison of the arterial patterning easier. The oldest arteries tend to progressively form regular boundaries, while distal parts are still more random and bushy. Note the completely spontaneous formation of a crablike pattern, the progressive extension of a stable circular sinus vein, with an angular re-entrance at the head. $\mathrm{SV}=$ sinus vein; $\mathrm{VA}=$ vitelline arteries.

ture which cannot be obtained by Kirchoff's law.

The vessels are more random at the distal tips and more regular in the proximal parts. The regularization process progresses in a proximal to distal order, just as observed in vivo. The physical explanation of this fact is simple: in the presence of line tension, a boundary which moves in a Laplacian field is stable for all wavelengths if the speed is negative with respect to the pressure gradient. In the vascular system, eventually the vessels do not move away, they only reorganize, so $V$ fluctuates around zero, but always with a $d V$ which is negative with respect to $\operatorname{grad} P$ (because a push always amounts to a recession, and not a growth). In addition, the line tension is very large, so all vessel strands ultimately smoothen. The formation of straight vascular filaments is analogous to the equilibration of boundaries which push each other. The process generates a pattern akin to a polygonal Dirichlet domain [25], as classically obtained when moving boundaries are pushed against each other, although here it is an open growing tree. This self-organization is then a consequence of concomitant adaptation of the vessels and of the interstitial tissue. Without the deformation of the vessels, one gets DLA-like vessels, which are too bushy [16]; this is also true of all models which are limited to variations in diameters of on-lattice vessels. In quasistatic growth, one will find the straight vessels aligned with the steepest gradient of pressure. Indeed, since the boundaries equilibrate pressure, there will be no pressure drop across them at equilibrium, so they are eventually perpendicular to isopressure lines. Seen otherwise, the strands are pushed by hits in the direction of tissue growth, which is the direction of $-\operatorname{grad} P$ in the solid. In general terms, the pattern of lines 

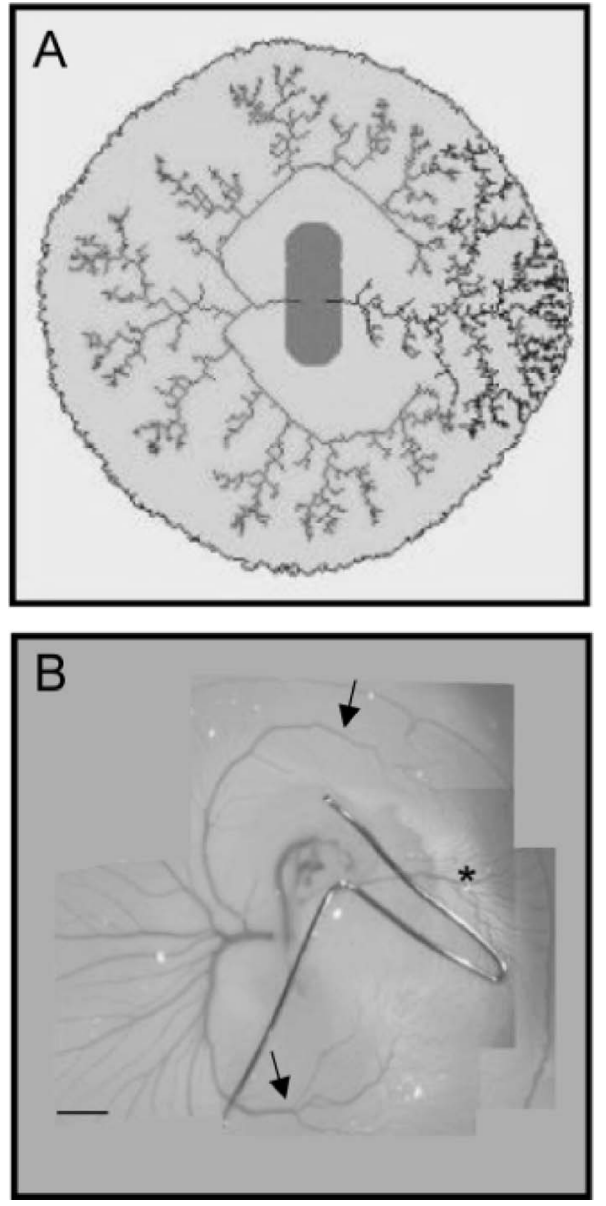

FIG. 15. (a) shows the calculated prediction of arterial occlusion on patterning by the physical model. The simulation was started with a single artery to the left and a single vein to the right. In this situation the arterial tree grows and tilts towards the right side of the embryo. Similar to the in vivo situation, two main branches pass the head-to-tail axis, one in the head region, one in the tail region. This situation amounts to transforming a quadrupole into a dipole. (b) shows the effect of a mechanical conclusion of the right vitelline artery on global patterning of the arteries and veins in the chickembryo yolk sac. Note in comparison to the control situation in Fig. 5 that the global patterning has changed for both arteries and veins. Arteries start to grow from the left-hand side towards the right-hand side, crossing the midline, moving towards the venous plexus on the right side. The right vitelline artery has transformed into a vein. Both the posterior (caudal) and anterior (cranial) vitelline veins are absent. Note the remains of the reentrance of the two cranial veins, along the sinus vein, in the region of the anterior ("north") pole (by 1 o'clock, in this image). Scale bar $1.5 \mathrm{~mm}$.

will tend to progressively follow the lines of steepest normal stress gradient.

The model, furthermore, allows predictions of the changes in arterial-venous vascular network structure based on changes in flow or tissue stress. We consider one example: the in vivo effect of arterial conclusion [Fig. 15(a)]. In this case the starting conditions for the corresponding simulation are a single artery on one side (loss of bilateral symmetry). The model predicts that the artery will split into two elongated loops crossing the head-to-tail axis. In- stead of having a quadrupolar shape as in the control, the boundary conditions are more of a horizontal bipolar nature (a flow output towards the yolk sac at the left side, a flow input from the yolk sac at the right). On the occluded side, a single vein forms which reaches out towards the sinus vein [Fig. 15(b)]. This is exactly what is observed in vivo $($ star $=$ vein, arrows $=$ artery $)$.

Similar simulations can be performed for other geometrical conditions such as the eye, or in the presence of twins, which will be presented elsewhere.

We have investigated the effect of a change of the values of the parameters in a simplified rectangular situation. Figure 16 shows typical morphologies, as a function of the parameters in the problem.

The interlace of arteries and veins in 3D is easily obtained as soon as crossing becomes possible. In proximal regions vessels do not flow directly into each other, since arteries are not connected. In distal parts, arteries and veins become interlaced, because of the interstitial tissue shear, which pushes veins towards the middle between existing arteries. This displacement is the sum of a considerable number of incremental displacements. With respect to a reference situation such as Fig. 16.1, the effect of varying the parameters is rather clear: increasing capillary disconnections distalizes the source of flow emanating from the tip arteries. This tends to generate long filamentous vessels; increasing the effect of compression by the interstitial tissue also favors elongated and sparse vessels.

\section{DISCUSSION AND CONCLUSION}

Taken together our data suggest that a dynamic interaction between the physical forces inside blood vessels due to flow of blood and the elastoviscoplastic solid tissue around collaborate in shaping the vascular architecture. Both vessels and interstitial tissue grow and adapt, but they should be considered as different, although interacting, components of the "living material," viewed as a composite. There is a true flow inside the vessels, and an elastoviscoplastic flow outside, in the tissue. This complex flow was modeled here as a viscous flow. Thin-film deformation of the cell layer of the yolk sac is akin to a Poiseuille flow. Interstitial "flow" affects vessel morphology, and conversely, vessel growth affects and feeds tissue growth. Alterations in this balance on either side may have profound effects on the establishment of the arterial-venous network in the tissue.

We furthermore demonstrate that a process of selforganization can result in the establishment of realistic branched arterial-venous networks, suggesting that branching of arterial networks does not have to be genetically hardwired. However, vessels tend to follow lines parallel to the local gradient of stress (pressure gradient lines, if the solid can be treated like an incompressible fluid).

The difference in mechanical properties may explain differences in vascular patterns between normal, hypertensive, or tumoral tissue, cartilage and bone, etc.

Capillary breakage is a new and most important feature of vessel formation. It blocks shunting between arteries and veins. As a result of vessel breakage, growing arteries can 

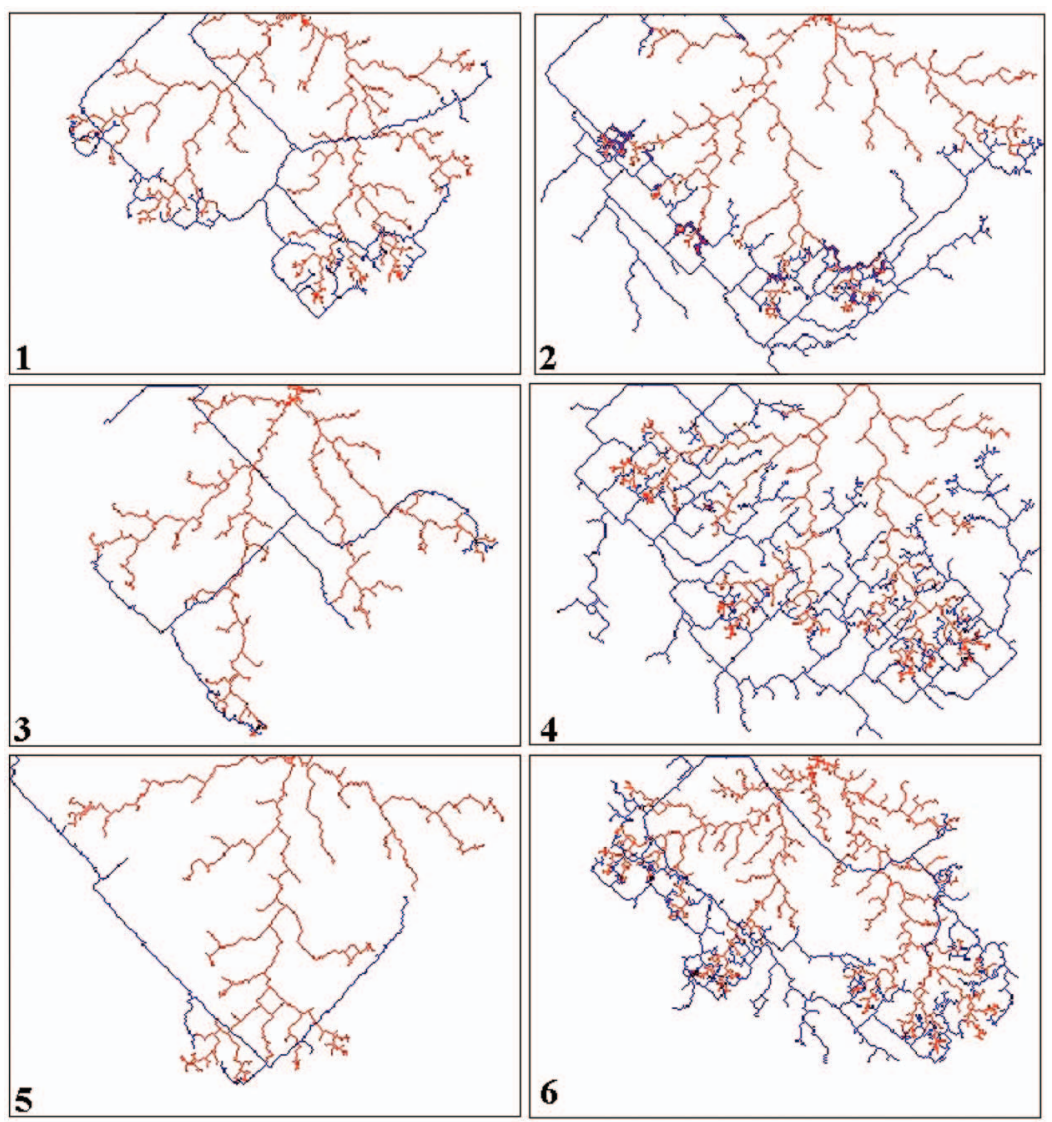

FIG. 16. (Color) Typical morphology diagram, as a function of parameters in the simulation. In this plate, we vary the following parameters: the proportion, $N_{\text {displace, }}$ of displacement-random walkers vs growth-random walkers. The proportion of disconnected vessels

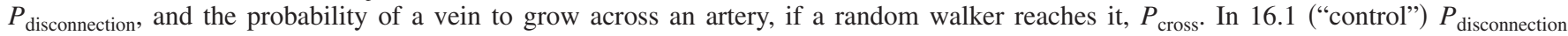
$=50 \%, N_{\text {displace }}=30, P_{\text {cross }}=1$, in $16.2 P_{\text {cross }}=0$, in $16.3 P_{\text {disconnection }}=80 \%$, in $16.4 P_{\text {disconnection }}=0$, in $16.5 N_{\text {displace }}=50 \%$, and in 16.6 $N_{\text {displace }}=10 \%$. In 16.1, a reference configuration in which the probability of crossing is one: veins cross arteries in the proximal regions without shunting and grow in direction of the tips of arteries (the sources of random walkers) where they interlace with arteries. In 16.2, the probability of crossing is now zero; as a consequence, veins are forced to turn around arteries to enter into the "fjords" in an interlace fashion. In 16.3, the capillary disconnection is enhanced; as a consequence, the sources of flow for the veins are more distal; this generates a pattern of very thin and elongated veins. In 16.6 the situation is shifted with respect to 25.1, towards less displacement; vessels are more bushy and exhibit direct $A-V$ shunts in the distal parts, because the deformation exerted by the interstitial tissue is not strong enough to keep the growing veins away from the arteries. In 25.5 we identify a set of ad hoc parameters which generates an interlace of arteries and veins. It is remarkable that the tendency of arteries to grow towards veins (and vice versa) may be equilibrated by the reaction of interstitial tissue which tends to shift them away; as a consequence, vessels approaching arteries tend to grow in parallel, with capillaries in between them (note that the capillaries are not shown, they form the discrete lattice on which the simulation is performed), as observed in the chick yolk sac, the chorioallantoic membrane, or even the retina vasculature. Such an interlaced vasculature is typical [1]. In 16.5, the physical push by interstitial tissue is very strong and capillary breakage is medium (50\% of the most distal arterial strands are still connected). Excess of capillary breakage and increased interstitial force appear as factors of vascular rarefaction, the major architectural feature of hypertension. Conversely, reduced capillary breakage or reduced interstitial force increases the number of $A-V$ shunts and makes more noisy, wavy, vessels. In simulations 2 and 3, the arteries were grown first and the veins were grown afterwards (as is the case for the secondary circulation stage). In all simulations, the total number of vein monomers which have been launched in order to grow a tree of a given size is bigger than for arteries. This comes from constant shifting and hindering of growth by displacement walkers and of discarded walkers when crossing is not possible. This is qualitatively correct in terms of tissue development: increased pressure or the presence of obstacles hinders growth. It is also an observed fact that veins grow more slowly than arteries, as appears in most images above. Remember that the final shape of a vascular bed is a result of progressive proximo-to-distal regularization of vessels by the self-organizing process; bushy venular beds become progressively elongated and thin strands, as commented above (especially Fig. 10; see also other figures).

carry blood to distal parts without loss. This permits the evolution of more distal appendages. As such, vessel breakage may, through regulation of arterial tree size, control organ development and allow the evolution of bigger animals.
Finally, we acknowledge that there exist other deformation modes than the one we have considered. Especially, vessel stretch from the inside of the vessel was not considered, nor were the buckling instabilities, which lead to meandering 
vessels whenever vessel elongation is faster than tissue growth. However, the experiments and models presented here open the way to a direct mechanical modeling of vasculatures. The challenge will be to integrate molecular and mechanical contributions to branching morphogenesis. Especially the complex mechanical behavior of endothelial cells, the interaction of blood flow with actin cytoskeleton rearrangements, and responses to growth factors may aid to generate artificial vessel or position new vessels in the context of therapeutic arteriogenesis [26].

\section{ACKNOWLEDGMENTS}

The finite-element calculations were done with the FREEFEM++ library developed by F. Hecht and O. Pironneau. We are grateful to $\mathrm{F}$. Hecht for providing the relevant routines and compiling libraries, http://www.freefem.org. F.1.N. was supported by a grant from the Royal Dutch Academy of Science (KNAW), Ter Meulen Foundation. V.F. was supported by the Microfluidics Program of the Centre National de la Recherche Scientifique and by the Fondation de France.
[1] A. L. Romanoff, The Avian Embryo (Macmillan, New York, 1960).

[2] S. Gilbert, Developmental Biology (Sinauer Associates, Sunderland, MA, 1994).

[3] V. Fleury, Organogenesis, 2, 1, 6 (2005).

[4] T. A. Witten and L. M. Sander, Phys. Rev. Lett. 47, 1400 (1981).

[5] V. Fleury, in Revue des questions scientifiques, Proceedings of the "Qu'est-ce que la vie?" symposium, edited by Guy Demortier (Presses de Facultés Notre-Dame de la Paix, in press).

[6] See this web site for a typical description of chick yolk-sac formation: http://www.swarthmore.edu/NatSci/sgilberl/ DB_lab/Chick/Chick_dev.html. There exist many other tutorial web sites.

[7] W. Risau, Nature (London) 386, 671 (1997).

[8] S. Isogai, N. D. Lawson, S. Torrealday, M. Horiguchi, and B. M. Weinstein, Development (Cambridge, U.K.) 130, 5281 (2003).

[9] F. Le Noble, D. Moyon, L. Pardanaud, L. Yuan, V. Djonov, R. Matthijsen, C. Bréant, V. Fleury, and A. Eichmann, Development (Cambridge, U.K.) 131, 361 (2004).

[10] R. Thoma, Untersuchunung über die Histogenese und Histomechanik des Gefässystems (Ferdinand Hencke Verlag, Stüttgart, 1893).
[11] V. Fleury, Phys. Rev. E 61, 4156 (2000).

[12] T. C. Skalak and R. J. Price, Microcirculation (Philadelphia) 3, 143 (1996).

[13] A. R. Pries, T. W. Secomb, and P. Gaehtgens, Am. J. Physiol. 275, H349 (1998).

[14] R. J. Price and T. C. Skalak, Microvasc. Res. 47, 188 (1994).

[15] R. J. Price and T. C. Skalak, Microcirculation (Philadelphia) 2, 41 (1995).

[16] D. G. Grier and D. Mueth, Phys. Rev. E 48, 3841 (1993).

[17] V. Fleury and L. Schwartz, Fractals 7, 33 (1999).

[18] V. Fleury and L. Schwartz, Fractals 8, 255 (2000).

[19] E. Guyon, J.-P. Hulin, and L. Petit, Hydrodynamique Physique (Editions du CNRS, Paris, 1991).

[20] L. Pietronero and H. J. Wiesmann, J. Stat. Phys. 36, 909 (1984).

[21] I. Stalmans et al., J. Clin. Invest. 109, 327 (2002).

[22] S. Claxton and M. Fruttiger, Exp. Eye Res. 77, 305 (2003).

[23] Jean-Pierre Marc-Vergnes (personal communication).

[24] T. Vicsek, Fractal Growth Phenomena (World Scientific, Singapore, 2000).

[25] P. Meakin, Fractals, Scaling and Growth Far from Equilibrium, Cambridge Nonlinear Science Series (Cambridge University Press, Cambridge, England, 1998).

[26] A. J. Koch and H. Meinhardt, Rev. Mod. Phys. 66, 1481 (1994). 